\title{
Dilution of compatibility effects in Simon-type tasks depends on categorical similarity between distractors and diluters
}

\author{
James D. Miles, Motonori Yamaguchi, ANd Robert W. Proctor \\ Purdue University, West Lafayette, Indiana
}

\begin{abstract}
The presence of a distracting stimulus during performance of the Stroop color-naming task leads to dilution of the Stroop effect. Because the automatic activation of word meaning may interfere with the task-relevant stimulus feature (text color; stimulus-stimulus $[\mathrm{S}-\mathrm{S}]$ interference) and the response (saying the text color; stimulus-response $[\mathrm{S}-\mathrm{R}]$ interference), it is unclear which of these types of interference is diluted. We introduce a new dilution paradigm using word- and arrow-based Simon tasks, in which only S-R interference is present. Participants made a left or right response to a central color target. A task-irrelevant location-word (Experiment 1) or arrow (Experiment 2) distractor adjacent to the target produced S-R compatibility effects. An additional neutral word or symbol series (diluter) was sometimes presented on the opposite side of the target from the distractor. The compatibility effect was smaller when the distractor and diluter category domains matched than when they mismatched. This result provides evidence that $\mathrm{S}-\mathrm{R}$ compatibility effects are susceptible to the presence of diluters that are categorically similar to the distractors.
\end{abstract}

Performance of choice reaction tasks is influenced by the features of task stimuli, even when those features are irrelevant to response selection. For example, in Stroop tasks, response times (RTs) are longer when task-relevant and -irrelevant stimulus features are incompatible with one another than when they are compatible (Stroop, 1935; see MacLeod, 1991, for a review). In the color-naming version of the Stroop task, this conflict on incompatible trials arises when the ink color is conveyed by a word that spells out an alternative color, such as when trying to name the ink color green in the presence of the word RED. The resulting increase in RTs and errors to words with incompatible colors and meanings, as compared with those with compatible colors and meanings, is referred to as the Stroop effect. Because the Stroop effect reflects the influence of a word's meaning that is irrelevant to performing the task, the Stroop effect is often taken as evidence that word reading is automatic (Keele, 1972; Posner \& Snyder, 1975).

\section{Stroop Dilution}

If word reading is truly automatic in the traditional sense, it must be involuntary and free from attentional demands. However, the idea that word reading occurs without the need for attention has been called into question by the phenomenon of Stroop dilution (Kahneman \& Chajczyk, 1983). In the most common Stroop dilution paradigm, participants are to respond to a target color bar by naming its color. In addition to the target stimulus, two other stimuli are presented concurrently: a distractor and a diluter. The distractor is a color word that may interfere with naming the color of the target, thereby leading to a Stroop effect. The diluter is an additional stimulus that does not interfere with color naming but may reduce the influence of the distractor. For example, when responding to the target bar by naming its color, participants' RTs are longer when the color word distractor and target bar indicate different colors rather than the same color. When a neutral word (e.g., talk) is presented simultaneously with the target and distractor, the Stroop effect is diminished, and Stroop dilution has occurred.

Brown, Roos-Gilbert, and Carr (1995) summarized three accounts of Stroop dilution. First, dilution may occur at the level of lexical processing. According to this account, Stroop dilution takes place because of a general capacity limit for word processing. When a color word distractor and neutral word diluter are presented concurrently, both words are processed in parallel. Since lexical processing is a limited resource, the meaning of each individual word is only partially activated. Assuming that there is a relation between the level of word activation and the word's influence on behavior, the effect of the distractor is reduced. Kahneman and Chajczyk (1983) tested this possibility using a neutral word or string of Xs as the diluter. If words are read automatically and dilution is a consequence of the finite resources of lexical processing, then the X diluter should not reduce the size of the Stroop effect, since it does not use this resource. However, the $\mathrm{X}$ diluter showed an amount of Stroop dilution that did not differ 
significantly from that produced by neutral words, suggesting that dilution occurs regardless of whether there is competition for lexical processing resources. Subsequent research confirmed this finding, showing that Stroop dilution also occurs with various other nonword, symbolic diluters. Most important, Brown et al. found that strings of different symbolic characters produced the same amount of Stroop dilution as did neutral words.

On the basis of this result, Brown et al. (1995) suggested a second account, that Stroop dilution is a function of visual complexity and occurs at an early visual processing stage, before lexical encoding. According to Brown et al.'s early visual interference account, all items in a visual display are processed in parallel. However, because general visual attention resources are limited, the presence of multiple visual stimuli degrades the feature representations that provide input to lexical memory. By this account, any diluter that accompanies the color word leads to reduction in the quality of the processing that the distractor word receives, reducing the Stroop effect. Thus, although increasing the visual complexity of a diluter may further reduce the influence of the color word distractor, the semantic or conceptual nature of the diluter is largely irrelevant.

Third, according to an attentional capture account, the distractor and diluter do not directly interfere with one another but are processed sequentially (Kahneman \& Chajczyk, 1983). When the distractor and diluter are presented concurrently, only one captures attention and is identified prior to response selection. Because there is an equal probability on a given trial of the distractor or diluter being processed, the size of the Stroop effect should be reduced by $50 \%$, which is what Kahneman and Chajczyk found.

Recently, researchers have modified the attentional capture account to include situations in which the allocation of attention to various items is not random but is instead biased, or privileged, to certain types of stimulus features. Wühr and Waszak (2003) showed that irrelevant color word distractors have much larger effects in the Stroop task when the distractor and color target are parts of the same object than when they are separate. Cho, Lien, and Proctor (2006) demonstrated that little Stroop effect occurs when the color target includes the diluter but that a large Stroop effect occurs when the color target includes the distractor, regardless of whether the color carrier is at fixation or at a peripheral location. Cho et al.'s (2006) results suggest that attention is directed initially to the color target stimulus even when it is not at fixation (see also Kim, Cho, Yamaguchi, \& Proctor, 2008). Taken together, these findings imply that dilution effects are sensitive to where attention is oriented.

On the basis of these studies, it is likely that Stroop effects are largest when most attentional resources are allocated toward the color word on the display. There is also evidence that these attentional resources are to some degree domain specific. For example, Roberts and Besner (2005) provided participants with a Stroop dilution task in which a diluter was always embedded in the color target and this target was flanked by a color word. In their Experiments 6-9, the diluter was a neutral word, a re- peating character (e.g., XXXX), nonletter symbols (e.g., "\%\&\#\}*"), or a series of numbers. Their results showed that the compatibility effect due to the color word was reduced only when the diluter was a neutral word. Roberts and Besner concluded that an important component of Stroop dilution is that the color word distractor and the diluter are from the same domain. Although their data provide support for this argument, this conjecture is currently limited to cases in which the distractor is a color word. To make a broader claim about the domain specificity of such attention effects, it is necessary to provide examples of domain-specific dilution effects in which the distractor is from a nonword domain. In such a case, a nonword diluter from the same domain as the distractor should lead to dilution of the compatibility effect and a neutral word diluter should have no effect.

\section{Simon Dilution}

Similar to the Stroop effect, compatibility effects in the Simon task occur when conflict exists between taskirrelevant information and features of the task response (for reviews, see Lu \& Proctor, 1995; Simon, 1990). For example, when a left or right response is to be made to the color of a red or green target presented on the left or right side of a display screen, responses are faster and more accurate when they match the task-irrelevant location of the stimulus than when they do not. Thus, the compatibility effect in the Simon task is defined as RT on spatially incompatible trials minus RT on spatially compatible trials. Of importance to the present study, this task-irrelevant spatial information can be conferred via several different stimulus modes in addition to physical location, including uppercase spatial words (e.g., LEFT, RIGHT) or left- and right-pointing arrows (for word- and arrow-based versions of the Simon task, see Miles \& Proctor, 2009; Proctor, Yamaguchi, Zhang, \& Vu, 2009). Regardless of how this spatial information is expressed, the important feature of the compatibility effect in the Simon task for the present study is that this information biases spatial responses, even though it is irrelevant and can be ignored when the specified task is successfully performed.

It is likely that the compatibility effect in the Simon task is susceptible to dilution in a way similar to that for the Stroop effect, but no study to date has examined this possibility. There is some debate as to whether the cognitive processes underlying compatibility effects in the Stroop and Simon tasks represent similar (O'Leary \& Barber, 1993) or different (Kornblum, 1994) cognitive phenomena. A fundamental difference between compatibility effects in the Stroop and Simon tasks is the relation of the irrelevant information to the relevant stimulus information and responses. As previously mentioned, the Stroop effect is due to the task-irrelevant word meaning, which may interfere with one's identifying the ink color of the word (stimulus-stimulus [S-S] compatibility) and cause one to respond by saying the ink color (stimulusresponse [S-R] compatibility). In contrast, the Simon task only contains an S-R compatibility effect between a taskirrelevant spatial feature and the spatial response to the task-relevant feature of a target stimulus. 
Neuroimaging research provides additional evidence of different cognitive processes at use in the Stroop and Simon tasks. Liu, Banich, Jacobson, and Tanabe (2004) found that the location-based Simon task led to greater activation in brain areas associated with motor control (the anterior cingulate cortex and supplementary motor areas) and areas associated with visuomotor transformation. In contrast, the Stroop task led to activation in the inferior parietal cortex, an area associated with biasing stimulus processing. These differences in activation suggest that the locus of compatibility effects is $\mathrm{S}-\mathrm{R}$ compatibility in the Simon task and mainly S-S compatibility in the Stroop task. It is noteworthy that a variation of the Simon task in which arrows conferred spatial information rather than physical location showed much greater similarity in neural activation between the tasks (Peterson et al., 2002), although the additional factors in their version of the Simon task may have contributed to this result (see Liu et al., 2004).

Kornblum (1994) suggested that S-S correspondence and S-R correspondence produce separate compatibility effects at different stages of processing: stimulus identification and response selection, respectively. He provided evidence for this claim by showing that, when participants performed a composite Stroop and Simon task, compatibility effects originating in both the S-S and S-R relations occurred and did not interact with one another. The distinction between S-S and S-R compatibility effects is especially relevant for the Stroop dilution paradigm. Because the color information that must be extracted from the target and the adjacent color word are conceptually similar, encoding the word may delay or interfere with target identification. In the Simon task with location words, the target color and irrelevant location word do not share any similarity that may lead to interference during identification. Because compatibility effects may occur as early as target identification in the Stroop color-naming task but not until response selection in the Simon task, the compatibility effect in the latter task may be less susceptible to dilution. This lessened susceptibility would be expected if the dilution effects occur primarily during the early processing stage of stimulus identification, rather than at the later stage of response selection. It is worth noting that, subsequent to Kornblum's research, Hommel (1997) contradicted a purely sequential model of processing stages for S-S and S-R compatibility effects and suggested that there is some temporal overlap between them. Even if these processes overlap in time, they may still be considered separable, with stimulus-processing stages more sensitive than response-selection processes to other stimulus information.

\section{The Present Experiments}

So far, Stroop dilution experiments have examined the influence of various types of diluters on the influence of a color word distractor. In the present study, we modified this paradigm to examine dilution of only S-R compatibility effects in word- and arrow-based Simon tasks. A Simon dilution paradigm has costs and benefits when compared with a Stroop dilution design. Stroop tasks lead to larger
RT differences between compatible and incompatible trials than do Simon tasks; this disparity makes it easier to detect differences, especially reductions, in compatibility effects in the former. However, although the compatibility effects in Stroop tasks are larger, it is difficult to determine the specific contributions of S-S and S-R compatibility to the overall size of the effect. Although smaller, the compatibility effects in Simon tasks can be attributed completely to S-R compatibility. Thus, the Simon task is a more precise tool for understanding the influence of the environment on response selection.

Furthermore, the compatibility effect in the Simon task relies only on task-irrelevant spatial information, which can be conferred in spatial locations, words, or symbols, whereas the Stroop task typically uses word stimuli. This property allows for the manipulation of features of both distractors and diluters that is not possible in the Stroop task. Using this technique, we are able to test the claim made by Roberts and Besner (2005) that dilution effects are sensitive to domain-specific cognitive processing loads. In particular, the influence of the spatial distractor on behavior should be reduced most when the diluter and distractor use the same processing resources. If this is so, the influence of a word distractor on response behavior should be weaker when it is accompanied by a word diluter than when it is accompanied by a symbol diluter. Conversely, the effect of a symbolic arrow distractor should be weaker when the distractor is presented with a symbolic diluter than when it is presented with a word diluter.

Our goals for the present research were twofold. First, we wanted to ascertain whether a Simon task is susceptible to dilution effects in a way similar to the Stroop task. As noted, this allows determination of whether only S-S compatibility effects are susceptible to dilution or whether S-R compatibility effects can be reduced through the use of a diluter stimulus. Second, we sought to determine whether different categories of spatial distractors (word and symbolic) are equally susceptible to the influence of diluters. Resolution of this issue would provide evidence as to whether the dilution effect has its basis in codes that depend on or are independent of stimulus category.

\section{EXPERIMENT 1}

The purpose of Experiment 1 was to replicate the dilution effect within a word-based Simon task for which the irrelevant location information was conveyed by the location words LEFT or RIGHT. In particular, we examined whether the addition of a diluter stimulus (neutral word or symbol string) would reduce the size of S-R compatibility effects in the word-based Simon task.

\section{Method}

Participants. Forty-eight undergraduate students ( 17 male, $31 \mathrm{fe}$ male; $17-27$ years of age) enrolled in an introduction to psychology course participated in return for experimental credits.

Apparatus and Stimuli. The experiment was written using E-Prime (Version 2.0) on PC-compatible machines running the Windows XP operating system. The stimuli were presented on a 17-in. VGA LCD monitor. Responses to the stimuli were made by press- 
ing the "Z" or " $\mathrm{M}$ " keys on a keyboard with the left or right index finger, respectively. Participants viewed the computer screen from a distance of approximately $60 \mathrm{~cm}$. The target stimulus was a centrally presented, red or green, solid rectangle that was $3 \mathrm{~cm}$ high and $11.5 \mathrm{~cm}$ wide. A location word distractor (LEFT or RIGHT), which was in uppercase and was $2 \mathrm{~cm}$ high and 6 or $7.5 \mathrm{~cm}$ wide, respectively, was presented $6.5 \mathrm{~cm}$ above or below the center of the target. Three diluter conditions were used: no diluter, symbol diluter, and word diluter. In the no-diluter condition, the location-word distractor always appeared by itself above or below the target. In the symbol diluter condition, a symbol diluter appeared on the screen concurrently with the distractor. Similar to those used by Mitterer, La Heij, and van der Heijden (2003, Experiment 1), the symbol diluters were nonletter symbol strings (i.e., those symbols selected by pressing shift and a number key on a standard keyboard: “\#\$\&(\%," "!\%\#@)," "\$\%!\#," and "\%@(\$." These symbol diluters had the same dimensions as the distractor ( $2 \mathrm{~cm}$ high, $6-7.5 \mathrm{~cm}$ wide) and appeared $6.5 \mathrm{~cm}$ above or below the center of the target on the opposite side from the distractor. In the word diluter condition, a neutral word appeared in addition to the distractor. Four word diluters (TALK, DRUG, BRICK, and HOUSE) were chosen, to match the number of letters in the distractors and to share minimum semantic similarity to directional words. The word diluters were in uppercase and matched the sizes of the distractors. Examples of the display for the three diluter conditions for Experiments 1 and 2 are shown in Figure 1.

Procedure. Participants performed two blocks of each of the diluter conditions for a total of six blocks of 96 trials each. Both blocks of a given diluter condition were always presented sequentially. The order in which the diluter conditions were presented was counterbalanced across participants. At the onset of the experiment, participants were provided with on-screen instructions to ignore everything but the target and about how to indicate responses to the target. The instructions were followed by 8 practice trials that included at least 1 trial of each of the three diluter types (none, symbol, word). Practice trials were excluded from the final analysis. Each trial began with an empty black screen for $1,200 \mathrm{msec}$, followed by a centrally presented fixation (white cross) for $500 \mathrm{msec}$, and then followed by another empty screen for $800 \mathrm{msec}$. The color target then appeared at the center of the screen simultaneously with the distractor, which was above or below the target. In the symbol and word diluter conditions, a diluter was also presented simultaneously above or below the target opposite the location-word. This display remained on the screen until a response was made. If an early response was made, a tone sounded, the words "You responded too early" appeared on the screen for 2,500 $\mathrm{msec}$, and the trial ended. If an incorrect response was made, the same tone sounded, and the word "Incorrect!" appeared on the screen for $1,000 \mathrm{msec}$.

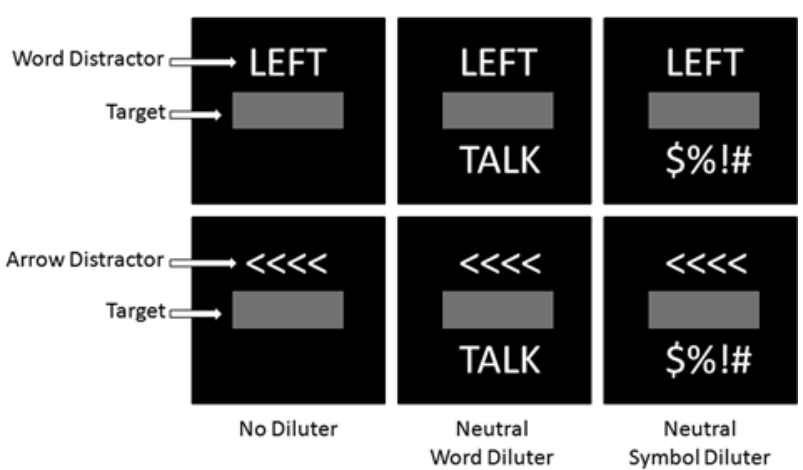

Figure 1. Example stimulus displays for Experiment 1 (word distractors, top row) and Experiment 2 (arrow distractors, bottom row) in each diluter condition. The target on each trial was either red or green.

\section{Results}

Figure 2 provides mean RTs and percent errors (PEs) for compatible and incompatible trials in each diluter condition. Trials with RTs shorter than $150 \mathrm{msec}$ were considered anticipatory and were removed from the analysis. RTs longer than 1,500 msec, which fell above the 99.9th percentile of the RT distribution in all conditions, were also removed to reduce the number of outliers. In total, $2.04 \%$ of trials were excluded from analysis. A $2 \times 3$ within-subjects ANOVA was run for the factors of compatibility (compatible vs. incompatible) and diluter condition (no diluter vs. symbol diluter vs. word diluter).

Of importance, a significant interaction was found for the compatibility $\times$ diluter condition $[F(2,94)=7.69$, $\left.M S_{\mathrm{e}}=359, p=.001\right]$. Pairwise $t$ test comparisons of each diluter condition showed that the compatibility effect was smaller with word diluters than with no diluter $[t(48)=$ $3.73, p=.001]$ or with symbol diluters $[t(48)=2.28$, $p=.03]$. Although the compatibility effect in the symbol diluter condition was smaller than that with no diluter, this reduction only tended toward significance $[t(48)=$ $1.81, p=.08]$. Overall, compatible trials $(M=532 \mathrm{msec})$ had a shorter mean RT than did incompatible trials $(M=$ $547 \mathrm{msec})\left[F(1,47)=18.70, M S_{\mathrm{e}}=843, p<.0005\right]$. The mean RTs for the no-diluter $(M=542 \mathrm{msec})$, word diluter $(M=530 \mathrm{msec})$, and arrow diluter $(M=546 \mathrm{msec})$ conditions were not significantly different from one another $(p>.30)$.

PE was low for every diluter condition and compatibility type (all PEs $<4.0 \%$ ). The main effect of compatibility reached significance, with fewer errors on compatible trials than on incompatible trials $[F(1,47)=24.32$, $\left.M S_{\mathrm{e}}=5.71, p<.0005\right]$. The diluter condition factor was also significant $\left[F(2,94)=5.78, M S_{\mathrm{e}}=4.67, p<\right.$ .01]. Within-subjects contrasts determined that PE was lower in the symbol diluter condition than in the conditions with no diluter $\left[F(1,47)=11.12, M S_{\mathrm{e}}=2.30, p<\right.$ $.01]$ or a word diluter $\left[F(1,47)=11.12, M S_{\mathrm{e}}=2.30\right.$, $p<.01]$. There was no difference in PE between the nodiluter and word diluter conditions $(p=.32)$. The diluter condition $\times$ compatibility interaction was not significant $[F(2,94)<1.0]$, and paired-sample $t$ tests of the compatibility effect in each diluter condition showed no differences (all $p s>.20)$.

\section{Discussion}

Even though the distractor and diluter are presented above and below the target in order to minimize locationbased compatibility effects, stimuli aligned along the vertical dimension can influence horizontally aligned responses differentially. Small compatibility effects $(<10 \mathrm{msec})$ have been reported in orthogonal Simon tasks, with a left response bias to a lower stimulus and a right response bias to an upper stimulus (Cho, Proctor, \& Yamaguchi, 2008; Nishimura \& Yokosawa, 2006). Therefore, we conducted the same ANOVA as above, including orthogonal compatibility (compatible, lower distractor-left response and upper distractor-right response; incompatible, upper distractor-left response and lower distractor- 

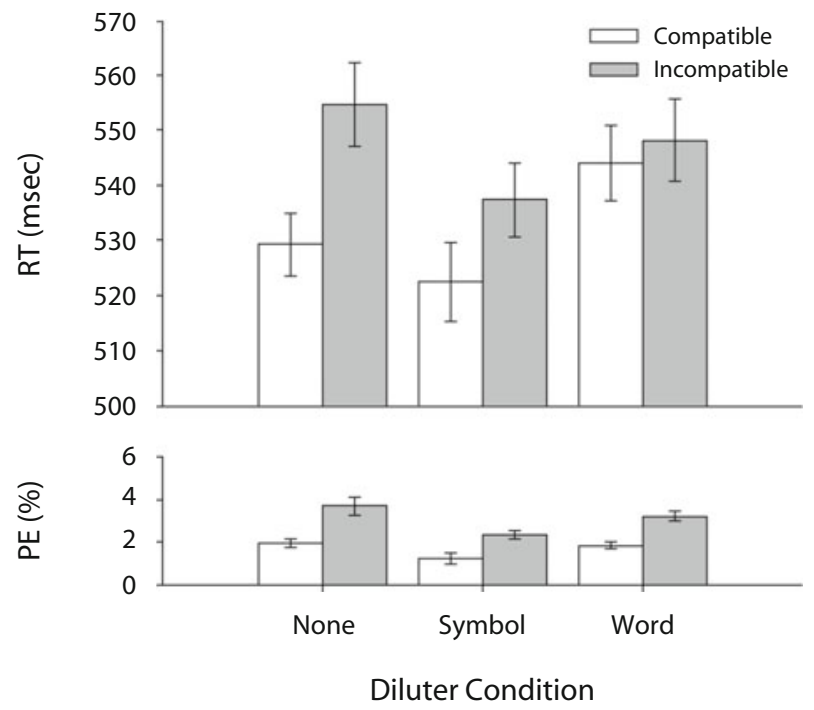

Figure 2. Mean response times (RTs) and percent errors (PEs), with within-subjects standard errors, for compatible and incompatible trials in each diluter condition of Experiment 1.

right response) as a factor. Orthogonal compatibility had no significant influence on performance and did not interact with diluter condition or compatibility between the distractor word and response (all $F \mathrm{~s}<1$ ). We therefore conclude that orthogonal Simon effects were not a critical factor in the present results.

Results show that, like the Stroop task, the word-based Simon task is susceptible to dilution effects. In particular, as compared with trials with no diluters, word diluters reduced the compatibility effect by $84 \%$ and symbol diluters by $36 \%$. The percentage of reduction in the compatibility effect caused by neutral word diluters was larger than that found in previous Stroop dilution studies, which usually ranged between $40 \%$ and $50 \%$. There are several reasons why dilution of this magnitude may have occurred in the present experiment. First, the size of the Simon task compatibility effect is smaller than that of the Stroop color-naming effect: Stroop effects without diluters range from $60 \mathrm{msec}$ to more than $100 \mathrm{msec}$ (e.g., Cho et al., 2006), whereas Simon task compatibility effects for location words are generally between 20 and $40 \mathrm{msec}$ (e.g., Miles \& Proctor, 2009; Proctor et al., 2009). This difference could partially reflect the fact that Simon-type stimuli have a weaker influence on behavior and are therefore relatively more sensitive to interference. Additionally, by definition, the targets and distractors in Stroop dilution experiments share a common conceptual feature, usually color, and this may partially protect the influence of the distractor from dilution. As described in the introduction, Cho et al. (2006) and Kim et al. (2008) found evidence that, in the Stroop dilution task, attention is biased to the item that carries the task-relevant target color. This bias may extend to conceptual properties as well: When the participant is searching for the color target, a color word may draw more attention than a neutral word does. When this conceptual similarity between the target and distrac- tor is removed, as in the present study, the distractor may become even more susceptible to dilution. In any case, we conclude that S-R compatibility effects in the word-based Simon task are susceptible to dilution in a way similar to that for compatibility effects in the Stroop task.

\section{EXPERIMENT 2}

In Experiment 2, we examined the Simon dilution effect when the task-irrelevant spatial information of the distractor was conferred symbolically by arrows rather than by word meaning. Each account of Stroop dilution described in the introduction leads to specific predictions for the resulting data. First, according to the lexical interference account, word diluters should not produce dilution of the compatibility effect, since the symbolic distractor does not rely on lexical processing resources. Second, the early visual interference account predicts that, as long as diluters are of equivalent visual complexity, they should lead to dilution of the compatibility effect. Because the distractors in Experiment 2 (arrows) may be considered less visually complex than those in Experiment 1 (location words), the diluters may produce an even greater reduction of the compatibility effect. According to an attentional capture account, the pattern of results should match those of Experiment 1, since distractors and diluters are processed serially and since the nature of these stimuli should be largely irrelevant to the capture of attention.

Additionally, the use of the arrow-based Simon task provides a critical test of Roberts and Besner's (2005) claim of domain-specific attentional resources. If, as they claim, dilution effects are stronger when the distractor and diluter are from the same processing domain and therefore share the same attentional resources, then the influence of the arrow distractor should not be affected by a neutral word diluter, and instead, the symbol diluters should lead to the greatest reduction in the compatibility effect.

\section{Method}

Participants. Forty-eight new undergraduate students (20 male, 28 female; $17-27$ years of age), from the same subject pool as that in Experiment 1 participated.

Apparatus and Stimuli. The design was similar to that in Experiment 1 . The main difference was that the location information of the distractor was conferred through a set of left- and right-pointing arrow symbols (either $<<<<$ or $>>>>$ ) instead of through location words. The arrow strings were $6 \mathrm{~cm}$ wide and $1.5 \mathrm{~cm}$ high. Since both the left and right arrow strings were of equal lengths, word and symbol diluters were all changed to be four characters long. The word diluters were talk, drug, cent, and hair, and the symbol diluters were “\#\$\&\%," “!\%\#@,”"\$\%!\#,” and “\%@(\$.” The height of all diluters was $2 \mathrm{~cm}$, and the width varied between 6 and $6.5 \mathrm{~cm}$.

Procedure. The experimental design was identical to that in Experiment 1 , except that the location information of the distractor was now conferred by a set of left- or right-pointing arrows rather than by word meaning. Once again, participants performed two blocks of 96 trials each for each of the three diluter conditions.

\section{Results}

Trials with RTs shorter than 150 msec or longer than $1,500 \mathrm{msec}$, which amounted to $1.80 \%$ of total trials, were excluded from analysis. A $2 \times 3$ within-subjects ANOVA 
was run for the factors of compatibility (compatible vs. incompatible) and diluter condition (no diluter vs. symbol diluter vs. word diluter).

RTs and PEs for compatible and incompatible trials in each dilution condition for Experiment 2 are shown in Figure 3 . The compatibility $\times$ diluter condition interaction did not reach significance $\left[F(2,94)=2.29, M S_{\mathrm{e}}=268\right.$, $p=.11$. However, pairwise comparisons of each diluter condition showed that, in a reversal from Experiment 1, the compatibility effect was $10 \mathrm{msec}$ smaller with a symbolic diluter than with a word diluter and this difference reached significance $[t(48)=2.12, p=.04]$. Unlike in Experiment 1, the compatibility effect was $5 \mathrm{msec}$ larger with a word diluter than with no diluter at all, although this difference did not reach significance $(p>30)$. As in Experiment 1, the symbolic diluter led to a small (5-msec) reduction in the compatibility effect that also did not reach significance $[t(48)=1.25, p>.20]$. Overall, RTs were shorter for compatible trials $(M=517 \mathrm{msec})$ than for incompatible trials $(M=538 \mathrm{msec})[F(1,47)=$ $\left.71.91, M S_{\mathrm{e}}=462, p<.001\right]$. There was no difference in RTs between the no- $(M=520 \mathrm{msec})$, symbol $(M=$ $532 \mathrm{msec})$, and word $(M=529 \mathrm{msec})$ diluter conditions $(p>.35)$. As in Experiment 1, an additional ANOVA showed that the orthogonal compatibility effect between distractor location and response location had no overall influence on RTs, nor did it interact with other factors (all $F \mathrm{~s}<1.9$, all $p \mathrm{~s}>.15$ ).

PE was low for every diluter condition and compatibility type (all PEs $<3.5 \%$ ). Overall compatible trial errors were fewer than incompatible trial errors $[F(1,47)=$ 14.67, $\left.M S_{\mathrm{e}}=11.04, p<.0005\right]$. A main effect was also found across diluter condition $\left[F(1,47)=4.71, M S_{\mathrm{e}}=\right.$ $2.57, p=.011]$. Within-subjects contrasts showed no difference between PE in the no-diluter and symbol diluter conditions $\left[F(1,47)=1.79, M S_{\mathrm{e}}=2.62, p=.19\right]$. PE
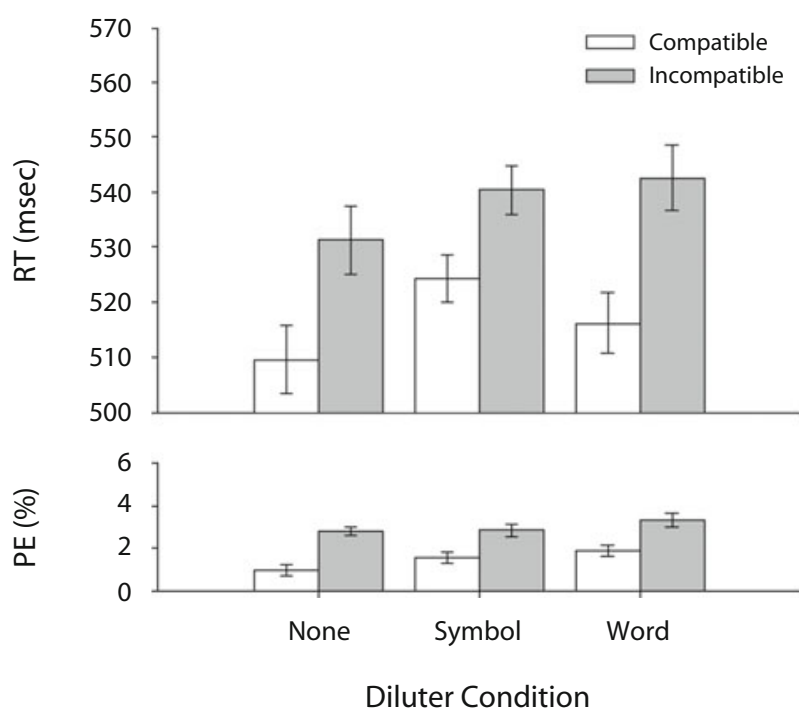

Figure 3. Mean response times (RTs) and percent errors (PEs), with within-subjects standard errors, for compatible and incompatible trials in each diluter condition of Experiment 2. in the no-diluter condition was significantly lower than that in the word diluter condition $\left[F(1,47)=8.88, M S_{\mathrm{e}}=\right.$ $2.71, p=.005]$, and PE was lower in the symbol condition than in the word diluter condition, but this did not reach significance $\left[F(1,47)=3.17, M S_{\mathrm{e}}=2.37, p=.08\right]$. A no-diluter condition $\times$ compatibility interaction was found $(p=.48)$. Paired-sample $t$ tests also showed no differences in the compatibility effect in individual diluter conditions (all $p$ s $>.20$ ).

\section{Discussion}

The size of the compatibility effect with no accompanying diluter was approximately the same for arrow distractors $(21 \mathrm{msec})$ in this experiment as for location-word distractors $(26 \mathrm{msec})$ in Experiment 1 . However, there was a different pattern of dilution in Experiment 2 than in Experiment 1. The word diluter now had no significant influence on the size of the compatibility effect. In fact, as compared with trials with no diluter, the compatibility effect increased by $19 \%$, although this difference was nonsignificant. The neutral word diluter greatly reduced the compatibility effect elicited by spatial word distractors but had no effect on the arrow distractor compatibility effect in the present experiment. As in Experiment 1, the symbol diluters had a nominal influence on the compatibility effect. Although symbol diluters reduced the arrow-based compatibility effect from 21 to $16(24 \%)$, this reduction was not significant.

Importantly, across Experiments 1 and 2, the compatibility effect was smaller when the distractor and diluter matched (word-word or symbol-symbol) than when they mismatched (word-symbol or symbol-word). To verify this observation, an across-experiment ANOVA was run, with similarity between the distractor and diluter (match vs. mismatch) and compatibility (compatible vs. incompatible) as within-subjects factors and experiment group (Experiment 1 vs. Experiment 2) as a between-subjects factor. The distractor-diluter similarity $\times$ compatibility interaction was significant $\left[F(1,94)=9.68, M S_{\mathrm{e}}=545\right.$, $p=.002]$, confirming that the compatibility effect was lower for a distractor-diluter match than for a distractordiluter mismatch. This result further supports Roberts and Besner's (2005) claim of domain specificity and provides evidence that nonword distractors are not influenced by word diluters.

It should be noted that this interaction was driven largely by the word diluter. The neutral word diluter eliminated the compatibility effect originating in the distractor words LEFT or RIGHT but had no effect on the influence of a left or right distractor arrow $\left[F(1,94)=11.69, M S_{\mathrm{e}}=699, p<\right.$ $.001]$. Previous studies showed that some amount of dilution occurs regardless of the nature of the diluter (Brown et al., 1995; Kahneman \& Chajczyk, 1983; Mitterer et al., 2003), but the present results indicate that the success of word diluters in reducing the compatibility effect is strongly dependent on the nature of the distractor that is the source of the compatibility effect. Alternatively, the symbol diluter produced only a small and nonsignificant reduction in the compatibility effect, regardless of whether this effect originated in a word or a symbol distractor 
$[F(1,94)<1.0]$. It is possible that the symbol diluter used in the present experiments was not similar enough to the arrow distractor to induce dilution of the compatibility effect. In particular, the arrow distractor was a set of repeating arrow characters, whereas the symbol diluter was a set of not repeating nonletter symbols. As previously pointed out by Roberts and Besner (2005), there is currently no systematic way to determine what types of distractors and diluters will share domain overlap; this point will be discussed further in the General Discussion.

\section{GENERAL DISCUSSION}

The present study extends dilution effects from the Stroop task to the Simon task. In the word-based Simon task, a word diluter reduced the compatibility effect produced by a location-word distractor but not that produced by a spatial symbol distractor. In contrast, a symbol diluter nonsignificantly reduced the compatibility effect to an equivalent degree, regardless of the distractor type. Importantly, this pattern suggests that Simon dilution is dependent on the relationship between the distractor that causes the compatibility effect and the type of diluter. Because the present experiments yielded a significant dilution only of the spatial word distractors, and not the arrow symbol distractors, a more conservative interpretation would be that the automatic processing of a diluter word interfered with the automatic processing of a word distractor, since the distractor required the same word-reading processes. Because symbolic arrow distractors did not require these word-reading processes, automatic processing of the arrows was not susceptible to the word diluters.

In any case, our results are at odds with a purely attentional capture account of dilution effects (Cho et al., 2006; Kahneman \& Chajczyk, 1983; Kim et al., 2008; van der Heijden, 1993). If attentional capture is a strictly serial process, then the categorical nature of the distractors and diluters should have little influence on the amount of dilution that occurs. As mentioned in the introduction, it is possible to bias the capture of attention by associating the distractor or diluter with task-relevant information or precued locations, but neither of these manipulations was performed in the present experiments. There is some evidence that the nature of the diluters in the Stroop dilution paradigm can bias attentional capture. However, this cannot explain the present results, since the task-relevant stimuli and responses did not change between Experiments 1 and 2. Consequently, the difference in Simon dilution cannot be attributed to a change in the relation between the task-relevant target and the distractor or diluter (e.g., Cho et al., 2006) or the predictability of the target, distractor, and diluter locations (Mitterer et al., 2003).

One might suggest that the reduction in the efficacy of word diluters from Experiments 1 to 2 is due to differences between the word and symbol distractors and that the categorical nature of the diluter is irrelevant. Remember, word distractors in Experiment 1 consisted of a relatively complex set of nonrepeating characters, whereas the symbol distractors were a series of repeating arrows. There are two possible ways that this difference could influence the effect of diluters in the Simon task. First, Brown et al. (1995) concluded that the magnitude of the Stroop dilution effect is at least partially determined by the visual complexity of the diluters, as compared with the distractors, with relatively more complex diluters leading to greater visual interference. Applying this logic to the present case, the complexity of the diluters relative to the distractors would have been less in Experiment 1 than in Experiment 2, since the distractors were words versus a single repeating symbol. Second, the arrow-based compatibility effects may be stronger and more robust than those of location words, as suggested by previous research showing that arrows sometimes produce larger compatibility effects than location words do (Proctor et al., 2009). Therefore, the word diluter was simply not as effective at interfering with the distractor in Experiment 2 as it was in Experiment 1.

The pattern of results obtained in the present study provides evidence that both of the above scenarios are unlikely. The size of the compatibility effect in the no-diluter condition was roughly the same in Experiments 1 and 2, suggesting that the location-word and spatial symbol distractors influenced response selection to an equivalent degree and were therefore of approximately equal strength. Therefore, it is unlikely that, as compared with word distractors, symbol distractors were simply more resilient against word diluters. More important, these explanations cannot account for the distractor-diluter similarity $\times$ compatibility interaction. Changing the perceptual complexity or strength of the diluter should lead to reductions or increases in the compatibility effect for all diluter conditions between Experiments 1 and 2. Although the compatibility effect with a word diluter increased from $4 \mathrm{msec}$ in Experiment 1 to $26 \mathrm{msec}$ in Experiment 2, it stayed at $16 \mathrm{msec}$ in both experiments with a symbol diluter.

There are two previously considered accounts of Stroop dilution effects that may explain the present results in the Simon dilution task. First, our results do not rule out a lexical interference account of dilution. The lexical interference account was rejected as an explanation for Stroop dilution because nonword diluters successfully led to reduction in the Stroop effect. This outcome suggested that, if interference occurs between items in a display, it is not at the level of lexical processing (Brown et al., 1995; Kahneman \& Chajczyk, 1983). However, in previous Stroop dilution research, the distractor stimulus was always a word, and only the nature of the diluter was varied. In Experiment 2 of the present study, the distractor was symbolic. In this case, according to the lexical account, a word diluter should not influence the compatibility effect, because lexical processing resources taken up by the diluter would not affect processing of a symbolic distractor.

A second, more precise explanation for the present results is similar to Mitterer et al.'s (2003) unlimitedcapacity attentional capture account of Stroop dilution, in which all stimuli in the display are processed in parallel to some degree. Additionally, we suggest that dilution occurs whenever there is competition for domain-specific processing resources, as suggested by Roberts and Besner (2005). This does not exclude the possibility of lexical 
interference, but rather suggests that lexical interference is merely one of several types of processing interference that can occur.

Several issues must be addressed when trying to understand the nature of the domain-specific effects in the present study. For example, considering that, at a surface level, both word and symbol diluters are character strings, why do word diluters have no effect on symbol distractors? A potential answer to this question is that processing conflicts do not occur until later processing stages. For instance, in the present experiments, after a distractor has been deeply processed and categorized as "word" or "string of symbols," it may interfere with spatial information within the same category. Multiple items may be identified more or less in parallel, as suggested by Mitterer et al. (2003), but interference may still occur when both items share categorical similarities and therefore require the same specific process for identification. In other words, there is no single stimulus-identification "bottleneck" but, rather, multiple separate processes associated with semantic identification. When a distractor and a diluter overlap in the same category, there is more competition for the same identification resources, and this leads to greater dilution of the compatibility effect. This idea is consistent with multiple-resource models of cognitive processes, in which categorically different types of representations, such as spatial and verbal codes, may be processed in parallel and similar codes lead to greater amounts of interference (Baddeley, 1986; Logie, 1995; Wickens, 1980; for a more recent review, see Wickens, 2002).

Evidence from previous research supports the possibility that semantic, or categorical, interference occurs in addition to, or without the need for, perceptual interference. Within the rapid serial visual presentation task, distractors with high conceptual similarity to a target are more likely to cause the target to be missed in the serial stream than are distractors with low conceptual similarity to the target, regardless of the similarity of their perceptual features (Chun \& Potter, 1995; Dux \& Coltheart, 2005; Isaak, Shapiro, \& Martin, 1999). Furthermore, previous research using intermixed trials of a location-relevant task (with incompatible mapping) and a location-irrelevant task (Simon task) provides evidence that the spatial representations produced by different stimulus modes are in part qualitatively different from one another. The effects on task performance produced when the location information for both tasks is in the same mode (e.g., arrows or location words for both tasks) are reduced when this information is in different categorical domains (Proctor, Marble, \& Vu, 2000; Vu $\&$ Proctor, 2002). Support for qualitatively different domains of spatial representation also comes from a method in which an incompatible mapping of a spatial stimulus to individual responses is practiced before the performance of a transfer Simon task. After 72 practice trials, complete transfer of the practiced mapping to the Simon task occurs when the categorical domain of the spatial information is physical position for one task and arrow direction for the other, but no transfer occurs when the categorical domain of the spatial information is physical location for one task and a location word for the other (Proctor et al., 2009). This pattern of results suggests that arrows and physical locations activate the same spatial representation but that words do not. Although relative perceptual complexity may play a role in dilution effects, as Brown et al. (1995) suggest, categorical similarity between the distractor and diluter is a deciding factor as well.

In conclusion, it is difficult to explain the present results in terms of a serial attentional capture account. Since the nature of the categorical relation between distractors and diluters modulates the magnitude of compatibility effects in the Simon task, parallel processing must occur to a sufficiently deep level to allow for the existence of domain-specific categorical knowledge of both stimuli. This conclusion is limited to the Simon task and S-R compatibility effects in the present study, and future research is needed to determine whether such categorical relationships play a similar role in dilution of S-S and S-R compatibility effects in the Stroop task as well.

\section{AUTHOR NOTE}

The research described in the present article was supported in part by ARO MURI Grant W911NF-05-1-0153. Address correspondence to J. D. Miles, Department of Psychological Sciences, Purdue University, 703 Third St., West Lafayette, IN 47907-1364 (e-mail: jmiles@, purdue.edu).

\section{REFERENCES}

Baddeley, A. D. (1986). Working memory. New York: Oxford University Press.

Brown, T. L., Roos-Gilbert, L., \& CARr, T. H. (1995). Automaticity and word perception: Evidence from Stroop and Stroop dilution effects. Journal of Experimental Psychology: Learning, Memory, \& Cognition, 21, 1395-1411.

Cho, Y.-S., Lien, M.-C., \& Proctor, R. W. (2006). Stroop dilution depends on the nature of the color carrier but not on its location. Journal of Experimental Psychology: Human Perception \& Performance, 32, 826-839.

Cho, Y.-S., Proctor, R. W., \& Yamaguchi, M. (2008). Influence of response position and hand posture on the orthogonal Simon effect. Quarterly Journal of Experimental Psychology, 61, 1020-1035.

Chun, M. M., \& PotTer. M. C. (1995). A two-stage model for multiple target detection in rapid serial visual presentation. Journal of Experimental Psychology: Human Perception \& Performance, 21, 109-127.

Dux, P. E., \& Coltheart, V. (2005). The meaning of the mask matters: Evidence of conceptual interference in the attentional blink. Psychological Science, 16, 775-779.

Hommel, B. (1997). Interactions between stimulus-stimulus congruence and stimulus-response compatibility. Psychological Research, 59, 248-260.

IsaAK, M. I., Shapiro, K. L., \& Martin, J. (1999). The attentional blink reflects retrieval competition among multiple rapid serial visual presentation items: Tests of an interference model. Journal of Experimental Psychology: Human Perception \& Performance, 25, 1774-1792.

Kahneman, D., \& ChaJCZYK, D. (1983). Tests of the automaticity of reading: Dilution of Stroop effects by color-irrelevant stimuli. Journal of Experimental Psychology: Human Perception \& Performance, 9, 497-509.

KeELE, S. W. (1972). Attention demands of memory retrieval. Journal of Experimental Psychology, 93, 245-248.

Kim, H., Сhо, Y. S., Yamaguchi, M., \& Proctor, R. W. (2008). Influence of color word availability on the Stroop color-naming effect. Perception \& Psychophysics, 70, 1540-1551. doi:10.3758/PP.70.8.1540

KornBLum, S. (1994). The way irrelevant dimensions are processed depends on what they overlap with: The case of Stroop- and Simon-like stimuli. Psychological Research, 56, 130-135.

Liu, X., Banich, M. T., Jacobson, B. L., \& Tanabe, J. L. (2004). 
Common and distinct neural substrates of attentional control in an integrated Simon and spatial Stroop task as assessed by event-related fMRI. Neurolmage, 22, 1097-1106.

LogIE, R. H. (1995). Visuo-spatial working memory. Hove, U.K.: Erlbaum.

Lu, C.-H., \& Proctor, R. W. (1995). The influence of irrelevant location information on performance: A review of the Simon and spatial Stroop effects. Psychonomic Bulletin \& Review, 2, 174-207.

MaCLEOD, C. M. (1991). Half a century of research on the Stroop effect: An integrative review. Psychological Bulletin, 109, 163-203.

Miles, J. D., \& Proctor, R. W. (2009). Reducing and restoring stimulus-response compatibility effects by decreasing the discriminability of location words. Acta Psychologica, 130, 95-102.

Mitterer, H., La Heij, W., \& van der Heijden, A. H. C. (2003). Stroop dilution but not word-processing dilution: Evidence for attention capture. Psychological Research, 67, 30-42.

Nishimura, A., \& YoKosawa, K. (2006). Orthogonal stimulus-response compatibility effects emerge even when the stimulus position is task-irrelevant. Quarterly Journal of Experimental Psychology, 59, 1021-1032.

O'Leary, M. J., \& Barber, P. J. (1993). Interference effects in the Stroop and Simon paradigms. Journal of Experimental Psychology: Human Perception \& Performance, 19, 830-844.

Peterson, B. S., Kane, M. J., Alexander, G. M., Lacadie, C., SkudLARSKI, P., LEUNG, H. C., ET AL. (2002). An event-related functional MRI study comparing interference effects in the Simon and Stroop tasks. Cognitive Brain Research, 13, 427-440.

Posner, M. I., \& SNyder, C. R. R. (1975). Attention and cognitive control. In R. L. Solso (Ed.), Information processing and cognition: The Loyola symposium (pp. 55-85). Hillsdale, NJ: Erlbaum.

Proctor, R. W., Marble, J. G., \& Vu, K.-P. L. (2000). Mixing incom- patibly mapped location-relevant trials with location-irrelevant trials: Effects of stimulus mode on the reverse Simon effect. Psychological Research, 64, 11-24.

Proctor, R. W., Yamaguchi, M., Zhang, Y., \& Vu, K.-P. L. (2009). Influence of visual stimulus mode on transfer of acquired spatial associations. Journal of Experimental Psychology: Learning, Memory, \& Cognition, 35, 434-445.

Roberts, M. A., \& BeSner, D. (2005). Stroop dilution revisited: Evidence for domain-specific, limited-capacity processing. Journal of Experimental Psychology: Human Perception \& Performance, 31, 3-13.

Simon, J. R. (1990). The effects of an irrelevant directional cue on human information processing. In R. W. Proctor \& T. G. Reeve (Eds.), Stimulus-response compatibility: An integrated perspective (pp. 3186). Amsterdam: North-Holland.

Stroop, J. R. (1935). Studies of interference in serial verbal reactions. Journal of Experimental Psychology, 18, 643-662.

VAN DER HeIJden, A. H. C. (1993). The role of position in object selection in vision. Psychological Research, 56, 44-58.

Vu, K.-P. L., \& Proctor, R. W. (2002). The prevalence effect for twodimensional S-R compatibility is a function of the relative salience of the dimensions. Perception \& Psychophysics, 64, 815-828.

Wickens, C. D. (1980). The structure of attentional resources. In R. S. Nickerson (Ed.), Attention and performance VIII (pp. 239-257). Hillsdale, NJ: Erlbaum.

WICKENS, C. D. (2002). Multiple resources and performance prediction. Theoretical Issues in Ergonomics Science, 3, 159-177.

WüHr, P., \& WASZAK, F. (2003). Object-based attentional selection can modulate the Stroop effect. Memory \& Cognition, 31, 983-994.

(Manuscript received December 17, 2008; revision accepted for publication May 17, 2009.) 